\title{
Supporting Situation Awareness of Dementia Patients in Outdoor Environments
}

\author{
Philipp Koldrack*, Ron Henkel ${ }^{\dagger}$, Frank Krüger ${ }^{\dagger}$, Stefan Teipel ${ }^{* \ddagger}$ and Thomas Kirste ${ }^{\dagger}$ \\ ${ }^{*}$ German Center for Neurodegenerative Diseases (DZNE), Rostock, Germany \\ $\dagger$ Department of Computer Science, University of Rostock, Rostock, Germany

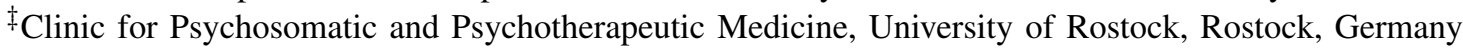

\begin{abstract}
Engagement in cognitively stimulating activities is today among the most effective measures against the decline of cognitive abilities due to dementing disorders. Outdoor and social activities are cognitively demanding and provide emotional support at the same time. Alzheimer's disease as the most frequent cause of the dementia syndrome, however, early effects skills for spatial orientation while it simultaneously impedes planning and error compensation abilities. These abilities are necessary to overcome disorientation in unfamiliar environments. People with dementia progressively limit their life-space and diversity of activities in reaction to growing security concerns. It is desirable to provide appropriate guidance in disoriented situations to avoid dangers, while not, in addition, complicating normal mobility in order to maintain a person's life-space, activity spectrum and cognitive health. Such guidance system has to be situation-aware and adaptive. Consequently, the mobility behavior of a patient must be modeled. In addition, the system must be able to learn and detect states of disorientation. In this paper we describe a pilot study to collect mobility data from dementia patients. Furthermore, we examine the collected data's quality and investigate how to detect states of disorientation within the data.
\end{abstract}

Keywords-dementia, alzheimer, sensor systems, assistive systems

\section{INTRODUCTION}

Interviewed about their perception of accessibility to public space, people with dementia reported, in a study of Brorsson et al. [1], an altered use of public space - a limitation to familiar activities and environments. The stated causes included motivational aspects, "people were no longer so fond of [...] adventures" [1, p.591]. But also feelings of vulnerability and insecurity due to "[...] experiences of lacking ability to stay in control when performing activities in the public space." [1, p.593] - were reported. This aligns with the observation of decreasing outdoor mobility [2], [3] and social activity [4], [5] in relation to progressive cognitive decline.

There is reason to believe, that regular cognitive stimulation has a protective effect on the mental health of people with dementia [6], [7], even if not on the pathological progress [8]. Outdoor and social activities provide a whole spectrum of cognitive stimulation, but with decreasing mobility, a major prerequisite for social contact gets lost [9], [10]. Isolation and accelerated cognitive decline are the likely result. Assistive technology for goal directed mobility, going beyond simple tracking and geofencing applications [11], has the potential to slow down this development if properly addressing the disease specific problems.
Lithfous et al. [12] provide a comprehensive overview of navigational skills and a comparison of performance between healthy aging and prodromal stages of alzheimer disease (AD). While performance in spatial tasks generally deteriorates with age, people with $\mathrm{AD}$ dementia show more severe impairments. This development already starts in the prodromal stage of mild cognitive impairment (MCI). Affected skills range from perceptual abilities over spatial learning to map-building abilities. On the perceptual layer, people with $\mathrm{AD}$ do not perceive their own motion as accurate as healthy controls on the basis of visual features. Keeping track of direction and distance moved is prone to errors. Recognizing landmarks is also an affected skill and reorientation therefore problematic. Navigating in known environments becomes a challenge, because the access to cognitive representations of spatial layouts is affected. Allocentric representations, referred to as cognitive maps, set spatial objects in relation to certain landmarks. This dynamic information structure enables younger people to recognize shortcuts, replan routes if a way appears to be impassable or to head toward not directly visible targets. This skill is impaired for elderly people, but heavily compromised in $\mathrm{AD}$ patients. The egocentric pendant recognizes environmental objects in relation to the own position and plays a major role in following well known path's. Perceived landmarks are mapped to learned responses, e.g. before the post-office turn left. Growing problems regarding this form of wayfinding, causes increasing difficulties, even when navigating along known routes.

Besides spatial orientation, goal-directed mobility requires preliminary planning and dynamic decision making, as information about the availability of routes or location of targets may be spatially distributed and is not directly accessible. This aspect of mobility was assessed by Passini [13] and Rainville [14]. Their study participants with AD were substantially less successful in formulating and executing plans on how to reach a specific target in an unfamiliar but topologically accessed public building. Confronted with an oversupply of information, e.g., when facing an information panel, study participants with AD experienced problems in limiting their attention to relevant aspects. Information that was accessible only in combination with memorized information or by inference was not usable for participants with AD. Simple direction pointers however were usually interpreted correctly. Also, the authors describe situations where certain events had triggeringeffects [13, p.828], for example nearby opening doors induced participants to step through for no discernible reason. Reaching a point were no information was available to guide the next steps, AD participants chose aimless walking or wandering in hope of reaching the desired target by chance, as leading 
strategy [14, p.59]. The author emphasizes the similarity to wandering behavior. Disorientation might therefore not only result from problems with perception or spatial cognition, but also with mislead decisions, attentional deficits and a tendency for spontaneous action.

The dismantling of distinct skills involved in goal-directed movement admits the healthy mobility-competent person to understand navigation in outdoor environments as the highly complex task it is. When Endsley writes about situation awareness (SA) in dynamic systems [15], he illustrates the proposed concept of SA with examples from the work of military pilots and power plant operators. However the concept and derived error taxonomy holds as well for wayfinding in the face of $\mathrm{AD}$. According to the author, expert-decision making in dynamic environments is most often based on a twofold process. At first, the expert acquires SA by eliciting the essential situational attributes. Then the pattern is matched to prototypical situations stored in the long-term memory. Elicitation of SA comprises three layers, the perception of environmental elements, the understanding of perceived elements in the context of current goals and the projection of future states of those elements. Working memory plays a key role in this process. It is necessary to direct attention at the perceptual layer and to further process and combine information on the upper layers. However, working memory is among the first affected cognitive skills in $\mathrm{AD}$, therefore $\mathrm{SA}$ will often be incomplete or erroneous. For example working memory enables a person to redirect attention to more relevant situational aspects, after attention was drawn by one highly salient but unimportant element. One could assume that a person with dementia, whose working memory is severely impeded, will often not be able to refocalize and irrelevant aspects become part of her SA, misguiding her decisions. The result may be scenes as observed by Passini [13], where a nearby opening door attracts the attention and the formerly pursued goal of reaching a specific target is no longer part of SA.

Assuming incomplete or erroneous SA due to disease specific perceptional and cognitive impairments to be the cause for undesired states in outdoor mobility, two concurring concepts of assistance present: (1) Providing comprehensible information in order that the user obtains more complete SA and can make better decisions. (2) Giving simple instructions, so the user has only to follow without straining cognition too much. We clearly favor the first approach, as keeping the person with $\mathrm{AD}$ active and independent is the actual motivation behind this work. Furthermore, arising technical problems or attention demanding environmental factors that can't be technically assessed, will likely put an aware person into less danger than somebody just following instructions.

\section{METHODS}

\section{A. Conceptual}

Our study is separated in a long time assessment of daily mobility (minimum of 4 weeks) and an accompanied 20 minutes walk along a predefined route of cognitively demanding and potentially stressful outdoor situations. Prior to the sensor based assessments participants and their caring relatives are interviewed on the topics of experience with, and attitude towards, technology usage and daily mobility. The long time assessment is recorded via GPS, accelerometry, and a mobility diary. Participants are asked to wear a bandage containing GPS and accelerometer every time they leave their home. In addition, for each trip they are asked to add an entry to their mobility diary, containing date, time, destination, stopovers, means of transport, incidents, and stating if the trip was accompanied. After approximately half of the planned time period the participant receives a personalized, smartphone based, navigation assistant to gather data on usage behavior and usability.

The accompanied walk takes place after the long time assessment. In addition to GPS and accelerometer, the participant is equipped with sensors to measure electrodermal activity and electrocardiography to conclude stress levels during the walk. For later analysis, the walk is videotaped. Prior to the recorded and videotaped part of the walk, the participant and a trained psychologist meet at a public location. ${ }^{1}$ The psychologist guides the participant to a tram station ${ }^{2}$ approximately one kilometer away. Aside from the usually crowded tram station, the route contains a four-way crossing of two main streets, as well as narrow streets in a residential neighborhood. The participant is asked to memorize the route. Starting from the tram station, the participant is asked to walk back on his own. On the way back the psychologist stays in the background, observes and protocols behavior and only interacts with the participant in case of unresolvable disorientation.

\section{B. Sensor Systems}

For our study we rely on different sensors. To record movements of our participants we use a GPS receiver (Qstarz Travel Recorder XT, BT747). From the available GPS sentences defined by the National Marine Electronics Association, we chose the recommended minimum (RMC) and essential fix data (GGA). We record Latitude, Longitude, Altitude, Speed, FIX, Date and Time using an interval of five seconds. For acceleration data we use the Move II activity sensor from Movisens recording the acceleration in three axes with $64 \mathrm{~Hz}$. Both sensors are placed in a bandage worn on the left ankle. For measuring the electrodermal activity of the skin we use the edaMove from Movisens $(32 \mathrm{~Hz})$, worn on the left wrist. A single channel electrocardiography is recorded by a Movisens ekgMove sensor $(256 \mathrm{~Hz})$, attached to the chest.

\section{Behavioral Model}

Computational Causal Behavior Models (CCBM) have been shown to effectively recognize goal oriented behavior [16], [17] from noisy or ambiguous sensor data. In order to provide situation awareness within our study we apply CCBM for activity and context recognition. A causal action model is created by means of precondition and effects. To later detect potential disorientation such states have to be included in the behavior model. A dynamic Bayesian network (DBN) is then synthesized from this model where nodes represent environment states and edges represent actions. The sensor model, necessary to infer user action, is created from annotated training data. Probabilistic filtering methods are then applied

\footnotetext{
${ }^{1}$ Cafe Paula, Rostock, Latitude 54.083431, Longitude 12.133500

${ }^{2}$ Doberaner Platz, Rostock, Latitude 54.088153, Longitude 12.123095
} 
to recognize the situation (incl. current activity, environment state and user goal).

\section{PILOT StUdy}

To develop a CCBM and train its sensor model, a pool of movement data and annotated behavior have to be available. In the following, we describe how to acquire such data, and provide an evaluation about its quality and usefulness.

We collected data sets from the long time assessment of two AD diagnosed participants (X001 and X002). A data set includes GPS and accelerometric data, points of interest defined by the participants and a mobility diary. To be able to use the collected data as a base for a CCBM and to identify potential occurrences of disorientation, it is necessary to evaluate the quality of the collected data and mobility diary. We thus evaluated if the mobility diary entries provided by the participants or their caretakers reflect the recorded GPS positions (Table I).

TABLE I. THIS TABLE STATES INFORMATION ABOUT THE ACCURACY OF THE MOBILITY DIARY (MD) AND THE RECORDED GPS DATA. AN ACTIVITY, IN THIS CASE, IS EVERY MOVEMENT AS STATED IN THE MD OR SHOWN BY GPS DATA IN A DISTANCE OF MORE THEN 200 METERS AWAY FROM THE PARTICIPANTS HOME. THE MOBILITY DIARY FOR X002 WAS WRITTEN BY THE PARTICIPANT'S CARING PARTNER.

\begin{tabular}{lll} 
Participant & X001 & X002 \\
\hline \hline Assessment duration (in days) & 35 & 41 \\
GPS recorded activities & 30 & 24 \\
Activities stated in MD & 84 & 33 \\
\hline Days without GPS data & 19 & 21 \\
Days without MD & 0 & 6 \\
\hline Activities stated in MD but no GPS data & 5 & 0 \\
Activities shown by GPS data but not stated in MD & 7 & 1 \\
Activities without time stated in MD & 3 & 1 \\
Activities in MD $\Delta>30 \mathrm{~min}$ & 9 & 8 \\
Activities stated accurately & 11 & 10
\end{tabular}

This evaluation shows that a significant amount of daily outdoor activities is not represented in the data, either because of missing MD entries or GPS data. Only 36\% (X001) and $41 \%$ (X002) of GPS recorded activities where stated accurately in the MD, meaning that a record exists and the start or end date is not off by more than 30 minutes. Furthermore, $13 \%$ (X001) and $30 \%$ (X002) of the activities stated in the MD are accurately shown in GPS data. As aforementioned, the participants were asked to state points of interest (POI). For the assessment duration the GPS data shows that X001 only visited one POI, X002 visited all except for one POI.

\section{DATA PROCESSING AND MOdELING}

In order to develop a CCBM able to recognize a participant's activities and detect states of disorientation, it is necessary to understand and annotate a participants behavior and movements. During the accompanied walk we actively try to invoke disorientation to be able to annotate such behavior and train the CCBM's sensor model. However, disorientation during the accompanied walk is not guaranteed. Thus, we also look for potential disorientation in our participant's long time assessment. As participants may not be aware of their own states of disorientation, we need means to recognize disorientation in our data and collect training samples. Figure 1 shows a map of recoded GPS positions, with color coded speed information, during a 15 minutes frame of an accompanied walk. The red colored records indicates spots worth further investigation. In addition, the local density of GPS records

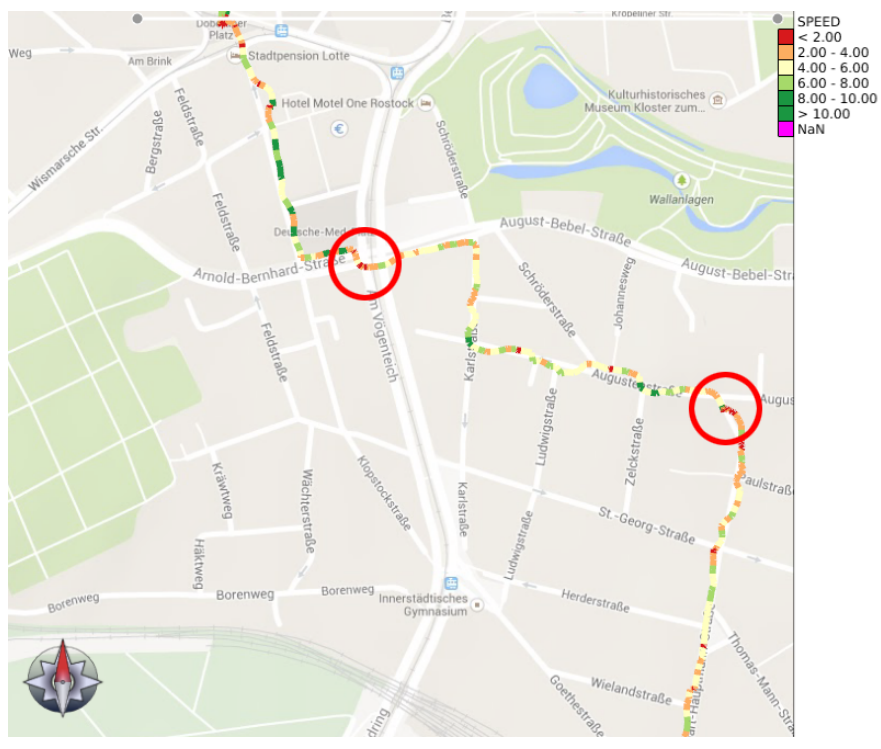

Fig. 1. Visualization of sample trajectory from a 20 min walk. The current speed is color coded, the legend shows the speed values in $\mathrm{km} / \mathrm{h}$. Areas with a high density of red and orange colored GPS records indicate potential disorientation. The red circles indicate locations of potential disorientation. Visualization is created with TrajectoryVis [18]

within a certain time frame can be used in a addition to the color coded map to describe disorientation such as wandering. For example, we highlighted a location (red circle, Figure 1 , center) showing the red colored records at the four-way crossing which indicates a waiting period due to traffic lights. If the time spent at this specific location increased further (the local density of recorded GPS positions increases), it would indicate a potential disorientation (e.g., problems crossing the street). A second location (red circle, right) also indicates potential disorientation. However, this location can not be explained by a crossing. As the data shown is taken from an accompanied walk, we know that the reason for the second location was the participant waiting for the accompanying person to catch up.

However, in order to annotate the data, a human curator familiar with the area the data sets were recorded, is inevitable. To ease annotation a video-stream is created showing the participants movements on the left and the according accelerometric data on the right (see Figure 2). The annotation contains information extracted from the mobility diary and identified states disorientation. In future, annotation together with the collected data will be used to develop a CCBM and train its sensor model.

Using GPS, accelerometric data and the mobility diary together, allows to distinguish movement patterns looking like disorientation from real disorientation. We will briefly outline a movement pattern we saw in the collected data that likely shows disorientation: The participant leaves her home, walks slowly on the sidewalk while stopping and waiting once in a while. In the following, the participant enters a wooded area and moves randomly within a small circle. She continues walking on the sidewalk and suddenly walks across a field. Even though this looks like disorientation, the mobility diary 


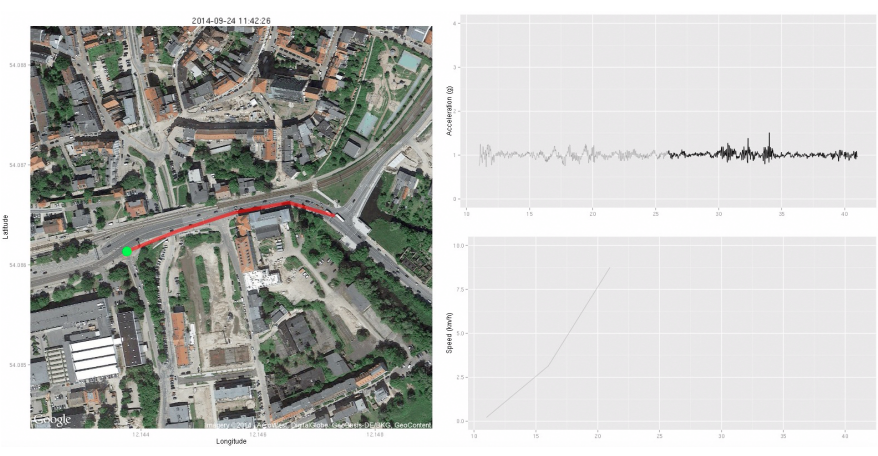

Fig. 2. Video created from collected GPS and acceleration data. On the left side, a part of the participant's movement is displayed on Google Maps. On the right side, information about the acceleration (top) and speed (bottom) is shown.

entry reveals that she was walking her dog. The same misleading conclusion might be drawn if a participant walks together with family members, e.g. grandchildren. This exemplifies how important a-priori knowledge will be for a system to recognize activities and intentions and explains why a CCBM must be aware of such participant depending situations.

\section{CONClusion}

To keep patients suffering from dementia mobile and thus healthy as long as possible is a valuable goal in an aging society. Today's possibilities of mobility support offers an effective alternative to the strategy of self-limitation of livespace. For the mobility support to be as less invasive as possible it is required to recognize activities, environment states and intentions as well as states of disorientation in a participants' mobility behavior. We aim at using Computational Causal Behavior Models (CCBM) for such recognition. In this manuscript we described the study design and results from two participants' long time assessments and accompanied walks. Subsequently, we explored the data gathered from our two pilot participants. We showed that the accuracy of matching activities with corresponding mobility diary entries is very low, in our case regardless if the diary was kept by the participant or the caretaker. Due to the low accuracy, data from the mobility diary is probably not suitable to be used without manual prior pre-processing.

In addition, we showed a concept how to identify locations of potential disorientation. Using knowledge about locations of potential disorientation allows us to investigate further, i.e. by talking to the participant in question, and validate or discard a disorientation assumption. We plan to annotate the datasets and use this knowledge to develop a CCBM and enable its sensor model to recognize participants' daily outdoor activities and potential states of disorientation. Such a CCBM may be able to observe a patient, recognize disorientation and offers help depending on what activity was interrupted by the disorientation. Altogether, such a system may increase a patients mobility, strengthen his self confidence, and, subsequently, improve the patients health and ease the work of the caretakeing family members.

While the pilot study described in this manuscript is completed, we aim to conduct the long term assessment and the accompanied walk with at least 12 additional AD participants.

\section{REFERENCES}

[1] A. Brorsson, A. Ohman, S. Lundberg, and L. Nygard, "Accessibility in public space as perceived by people with Alzheimer's disease," Dementia, vol. 10, no. 4, pp. 587-602, 2011.

[2] J. Kaye, N. Matteck, T. Hayes, D. Austin, and H. Dodge, "Community life withdrawal with mci progression," Alzheimer's \& dementia : the journal of the Alzheimer's Association, vol. 8, no. 4, p. P483, 2012. [Online]. Available: http://linkinghub.elsevier.com/retrieve/pii/S1552526012014409?showall=true

[3] M. Wettstein, H.-W. Wahl, N. Shoval, F. Oswald, E. Voss, U. Seidl, L. Frolich, G. Auslander, J. Heinik, and R. Landau, "Out-of-home behavior and cognitive impairment in older adults: Findings of the sentra project," Journal of Applied Gerontology, 2012.

[4] T. F. Hughes, J. D. Flatt, B. Fu, C.-C. H. Chang, and M. Ganguli, "Engagement in social activities and progression from mild to severe cognitive impairment: the myhat study," International Psychogeriatrics, vol. 25, no. 04, pp. 587-595, 2013.

[5] T. A. Glass, C. M. d. Leon, R. A. Marottoli, and L. F. Berkman, "Population based study of social and productive activities as predictors of survival among elderly americans," BMJ: British Medical Journal, vol. 319, no. 7208, p. 478, 1999.

[6] B. Woods, E. Aguirre, A. E. Spector, and M. Orrell, "Cognitive stimulation to improve cognitive functioning in people with dementia," Cochrane database of systematic reviews (Online), vol. 2, p. CD005562, 2012.

[7] M. Prince, E. Albanese, M. Guerchet, and M. Prina, World Alzheimer Report 2014: Dementia and Risk Recuction - An Analysis of protective and modifiable factors, Alzheimer's Disease International (ADI), Ed., 2014.

[8] D. A. Bennett, "Postmortem indices linking risk factors to cognition: results from the religious order study and the memory and aging project," Alzheimer disease and associated disorders, vol. 20, p. S63, 2006.

[9] H. Mollenkopf, F. Marcellini, I. Ruoppila, P. Flaschenträger, C. Gagliardi, and L. Spazzafumo, "Outdoor mobility and social relationships of elderly people," Archives of Gerontology and Geriatrics, vol. 24, no. 3, pp. 295-310, 1997.

[10] A. L. Rosso, J. A. Taylor, L. P. Tabb, and Y. L. Michael, "Mobility, disability, and social engagement in older adults," Journal of Aging and Health, vol. 25, no. 4, pp. 617-637, 2013.

[11] V. Faucounau, M. Riguet, G. Orvoen, A. Lacombe, V. Rialle, J. Extra, and A.-S. Rigaud, "Electronic tracking system and wandering in Alzheimer's disease: A case study," Annals of Physical and Rehabilitation Medicine, vol. 52, no. 7, pp. 579-587, 2009. [Online]. Available: http://www.sciencedirect.com/science/article/pii/S1877065709001766

[12] S. Lithfous, A. Dufour, and O. Després, "Spatial navigation in normal aging and the prodromal stage of alzheimer's disease: Insights from imaging and behavioral studies," Ageing Research Reviews, vol. 12, no. 1, pp. 201-213, 2013.

[13] R. Passini, C. Rainville, N. Marchand, and Y. Joanette, "Wayfinding in dementia of the alzheimer type: Planning abilities," Journal of Clinical and Experimental Neuropsychology, vol. 17, no. 6, pp. 820-832, 1995.

[14] C. Rainville, "A multiple case study of wayfinding in dementia of the Alzheimer type: Decision making," Aging, neuropsychology and cognition, vol. 8, no. 1, p. 54, 2001.

[15] M. R. Endsley, "Toward a theory of situation awareness in dynamic systems," Human Factors: The Journal of the Human Factors and Ergonomics Society, vol. 37, no. 1, pp. 32-64, 1995.

[16] F. Krüger, K. Yordanova, C. Burghardt, and T. Kirste, "Towards creating assistive software by employing human behavior models," Journal of Ambient Intelligence and Smart Environments, vol. 4, no. 3, pp. 209226, May 2012.

[17] F. Krüger, M. Nyolt, K. Yordanova, A. Hein, and T. Kirste, "Computational state space models for activity and intention recognition. a feasibility study." PLOS ONE, Nov. 2014.

[18] C. Tominski, H. Schumann, G. Andrienko, and N. Andrienko, "Stacking-Based Visualization of Trajectory Attribute Data," IEEE Transactions on Visualization and Computer Graphics, vol. 18, no. 12, pp. 2565-2574, 2012. [Online]. Available: http://dx.doi.org/10.1109/TVCG.2012.265 